Rev Biomed 2004; 15:207-213.

\title{
Prevalencia del intento de suicidio en el Servicio de Urgencias del Hospital General “Dr. Agustín O'Horán”, de enero de 1998 a diciembre de 2003.
}

\section{Artículo Original}

Soledad Coronado-Santos ${ }^{1}$, Ileana Díaz-Cervera ${ }^{1}$, Lizardo Vargas-Ancona ${ }^{1}$, Arsenio Rosado-Franco ${ }^{1}$, Alicia Zapata-Peraza $^{2}$, Silvia Díaz-Talavera ${ }^{1}$.

${ }^{1}$ Fundación Mexicana para la Salud - Capítulo Peninsular - Hospital "Dr. Agustín O’Horán, ${ }^{2}$ Centro de Investigaciones Regionales “Dr. Hideyo Noguchi”, Universidad Autónoma de Yucatán, Mérida, Yucatán, México.

\section{RESUMEN.}

Introducción. El suicidio es el acto encaminado a la autodestrucción intencional. El aumento en la tasa de suicido a nivel nacional plantea un verdadero problema de salud pública porque afecta a la población en edad productiva, siendo necesario implementar un programa preventivo para dicha situación.

Material y método. Se realizó un registro de libretas de trabajo social de los reportes hechos al ministerio público, así como de los expedientes de todos los pacientes mayores de 10 años, que acudieron al servicio de urgencias del Hospital General O’Horán por intento de suicido de 1998 al 2003.

Resultados. Se obtuvo un total de consultas atendidas de 1998 a 2003 de 713, de las cuales el $47.4 \%(\mathrm{n}=338)$ corresponden al sexo masculino y $52.6 \%(\mathrm{n}=375)$ al sexo femenino. El intervalo de edad más afectado es de 15 a 24 años. No se observó distribución específica por mes del año. En la mayoría de los casos el mecanismo utilizado para el intento de suicidio no se reportó. El 3.5 \% falleció a consecuencia de dicho intento.

Comentarios. El intento de suicidio es un motivo frecuente de consulta en el servicio de urgencias del Hospital General O’Horán. Se observó que existe un subregistro hospitalario al respecto, ya que tanto los intentos de suicidio como el suicidio son competencia del ministerio público, por lo que no fue posible obtener todos los datos requeridos para un análisis más completo. (Rev Biomed 2004; 15:207-213)

Palabras clave: Suicidio, epidemiología, Yucatán México.

\section{SUMMARY.}

Prevalence of suicide attempts in the Emergency Ward of the General Hospital "Dr. Agustin O’Horan” from January 1998 to December 2003. Introduction. Suicide is an act directed at intentional self-destruction. An increase in the national suicide rate

Solicitud de sobretiros: M.C. Soledad Coronado-Santos, Calle 107 Número 317 X 42 y 46 Col. Santa Rosa, C. P. 97279, Mérida, Yucatán, México. E-mail:wil35@hotmail.com jensenanza@prodigy.net.mx

Recibido el 1/Abril/2004. Aceptado para publicación el 18/Octubre/2004.

Este artículo está disponible en http://www.uady.mx/sitios/biomedic/revbiomed/pdf/rb041542.pdf

Vol.15/No.4/Octubre-Diciembre, 2004 
S Coronado-Santos, I Diaz-Cervera, L Vargas-Ancona, A Rosado-Franco, A Zapata-Peraza y col.

presents a seriuos public health problem because it affects the population at a productive age, raising the necesity of implementing a preventative program to address this situation.

Materials and Methods. The reports made to the Department of the Public Prosecutor from the social workers' records and from the files of all the patients over 10 years old, who attended the Emergency Ward at the O'Horan General Hospital for attempted suicide from 1998 to 2003 were registered.

Results. A total of 713 patients were attended between 1998 and 2003. 47.4\% $(\mathrm{n}=338)$ were male and $52.6 \%(\mathrm{n}=375)$ were female. The 15 to 24 age group ws the most affected. No specific distribution by month or year was observed. The mechanism used in the suicide attempts were not reported. 3.5\% died as a consequence of the attempt.

Comments. Attempted suicide is a frequent reason for visiting the Emergency Ward at the O'Horan General Hospital. There is a hospital sub-register on the subject since suicide and suicide attempts concern the Public Prosecutor which make it impossible to obtain all the required data for a more complete analysis. (Rev Biomed 2004; 15:207-213)

Key words: suicide, epidemiology, Yuacatan Mexico.

\section{INTRODUCCIÓN.}

La palabra suicidio proviene del latín "sui” que significa "uno mismo" y de "cidium" que se refiere a "Caédere” y se traduce como "matar", es decir, el acto del individuo encaminado a la autodestrucción intencional.

La Organización Mundial de la Salud (OMS), en 1996, se refiere al acto suicida como la lesión a sí mismo con diversos grados de intención mortal y de conciencia del motivo. Suicidio es entonces una autoagresión con resultados mortales. De este concepto se derivan los siguientes tipos de suicidio (1):

1. El consumado: que se refiere al hecho de una persona que atenta contra su vida y muere.

2. El intento de suicidio: hace referencia al hecho de vivir una conducta autodestructiva pero no morir. 3. El suicidio frustrado: se trata del momento en que una persona toma la decisión de quitarse la vida, pero en última instancia busca ayuda, con la intención de salir adelante.

4. El gesto suicida: este concepto nos habla de señales físicas o verbales, que da una persona y que en conjunto establece una probabilidad de llegar a atentar contra su vida.

El suicidio ha existido en todas la sociedades y épocas de la humanidad. Se le han atribuido diferentes causas, como ser el último acto de una persona libre, un acto heroico y hasta un pecado (2).

Actualmente la mayoría de los investigadores de las sociedades occidentales, consideran que el suicidio y el intento del mismo es un fenómeno complejo con factores psicológicos, biológicos y sociales asociados.

Las conductas suicidas constituyen uno de los problemas más importantes de salud pública en todos los países; la magnitud del mismo queda bien ilustrada por medio de las estadísticas de la OMS. En una revisión de 201 países publicada en 1900, pudo apreciarse como aproximadamente 808,000 personas se suicidaban al año en el mundo, lo cual supone un número similar al de personas fallecidas en accidentes de tráfico $(856,000)$ y superior al número de defunciones causadas por conflictos bélicos $(322,000)$ durante idéntico período. Sin embargo, estas cifras sólo representan la punta de un iceberg según datos de la OMS; aproximadamente 10.2 millones de personas en todo el mundo cometen algún tipo de conducta suicida que requiere atención médica y cerca de 30 millones estarían en peligro de cometer este tipo de comportamientos aunque no reclamen atención. A esto habría que añadir que aproximadamente 4.25 millones de personas se ven directamente afectadas por la muerte debidas a suicidio $(3,4)$.

El suicidio se encuentra entre el segundo y tercer lugar como causa de muerte en jóvenes de 15 a 19 años de edad y su incidencia se ha triplicado en los países occidentales en los últimos 30 años, especialmente en adolescentes y adultos jóvenes (7). Con mucha frecuencia entre los suicidas se encuentran

\section{Revista Biomédica}


Prevalencia del intento de suicidio en el Servicio de Urgencias.

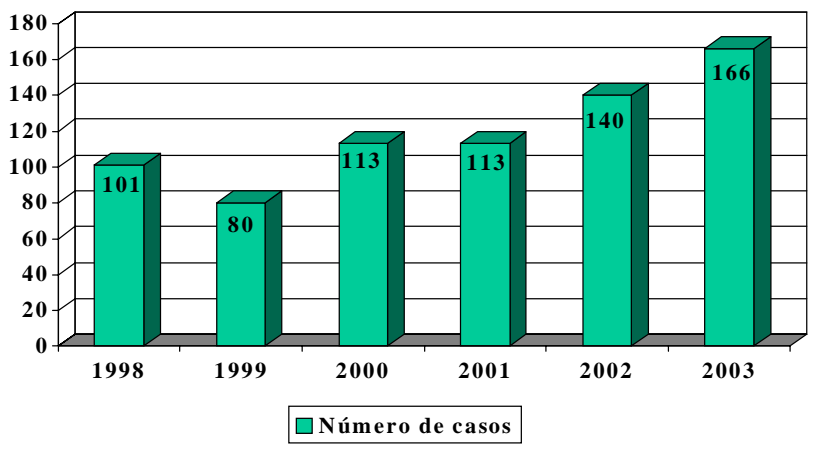

Figura 1.- Total de intentos de suicidio registrados de 1998 al 2003 en el Hospital General "Dr. Agustín O'Horán", Mérida, Yucatán, México.

alteraciones de la personalidad, estado de ánimo inestable, agresividad, impulsividad y alienación social. Cuando éstas se combinan con abuso del alcohol y drogas aumenta el riesgo de suicidio.

El presente trabajo se realizó con el fin de conocer las características demográficas (edad, sexo, estado civil, localidad, escolaridad) de las personas que intentaron suicidarse y que acudieron al Hospital General "Dr. Agustín O’Horán” de la ciudad de Mérida, Yucatán, México, así como establecer el método que utilizaron con más frecuencia, la comorbilidad psiquiátrica y el riesgo que presentan para intentarlo nuevamente.

\section{MATERIAL Y MÉTODO.}

Se realizó un estudio descriptivo, retrospectivo y transversal. Se indujeron a todos los pacientes mayores de 10 años, que fueron atendidos en el

\section{Cuadro 1}

Pacientes atendidos en Urgencias del Hospital General “Agustín O’Horán” de la Secretaria de

Salud, en Mérida, Yucatán, México y su relación con intento de suicidio.

\begin{tabular}{lccc}
\hline AÑO & $\begin{array}{c}\text { Total } \\
\text { atendidos }\end{array}$ & $\begin{array}{c}\mathbf{N}^{\mathbf{0}} \text { con intento } \\
\text { suicidio }\end{array}$ & \% del total \\
\hline 1999 & $\mathbf{8 5 6 6}$ & $\mathbf{8 0}$ & $\mathbf{0 . 9 3 \%}$ \\
2001 & $\mathbf{5 9 9 4}$ & $\mathbf{1 1 3}$ & $\mathbf{1 . 8 8 \%}$ \\
2002 & $\mathbf{7 2 3 2}$ & $\mathbf{1 4 0}$ & $\mathbf{1 . 9 3 \%}$ \\
2003 & $\mathbf{7 0 3 4}$ & $\mathbf{1 6 6}$ & $\mathbf{2 . 3 5 \%}$ \\
\hline
\end{tabular}

\section{Cuadro 2}

Distribución de intento de suicidio por sexo, de 1998 a 2003 en el Hospital General "Agustín O’Horán” de la Secretaria de Salud, en Mérida, Yucatán, México.

\begin{tabular}{llll}
\hline Año & Masculino & Femenino & Total \\
\hline 1998 & $43(43 \%)$ & $58(57 \%)$ & 101 \\
1999 & $39(48.8 \%)$ & $41(51.2 \%)$ & 80 \\
2000 & $55(48.7 \%)$ & $58(51.3 \%)$ & 113 \\
2001 & $59(52.2 \%)$ & $54(47.8 \%)$ & 113 \\
2002 & $69(49.3 \%)$ & $71(50.7 \%)$ & 140 \\
2003 & $73(44 \%)$ & $93(56 \%)$ & 166 \\
Total & $338(47.4 \%)$ & $375(52.6 \%)$ & $713(100 \%)$ \\
\hline
\end{tabular}

servicio de urgencias del Hospital General "Dr. Agustín O’Horán" de la Secretaria de Salud, en Mérida, Yucatán, México, por intento de suicidio, en los años de 1998 a 2003.

Se tomaron datos demográficos y clínicos de las libretas de registro de trabajo social, de los reportes hechos al ministerio público, así como de los expedientes. Estos datos fueron analizados con el paquete estadístico SPSS versión 10 para Windows.

\section{RESULTADOS.}

Se obtuvo un total de 713 consultas atendidas por intento de suicidio de 1998 a 2003, distribuidas de la siguiente manera: en 1998 se registraron 101 $(14.3 \%)$ intentos de suicidio, $80(11.2 \%)$ en 1999 ; $113(15.8 \%)$ en $2000 ; 113(15.8 \%)$ en $2001 ; 140$

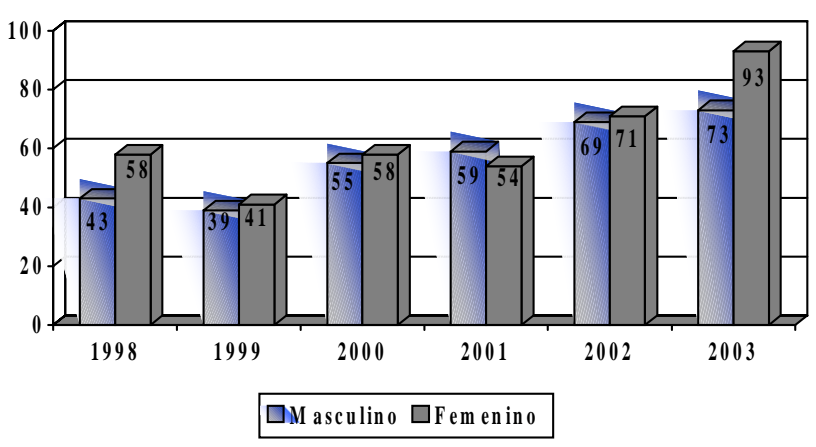

Figura 2.- Intento de suicidio por género de 1998 a 2003 registrados en el Hospital General "Dr. Agustín O'Horán", Mérida, Yucatán, México.

Vol.15/No.4/Octubre-Diciembre, 2004 
S Coronado-Santos, I Diaz-Cervera, L Vargas-Ancona, A Rosado-Franco, A Zapata-Peraza y col.

Cuadro 3.

Número de intentos de suicidio por género, en grupos de edad, en el Hospital General “Agustín O’Horán” de la Secretaria de Salud, en Mérida, Yucatán, México de 1998 a 2003.

\begin{tabular}{|c|c|c|c|c|c|c|c|c|c|c|c|c|}
\hline \multicolumn{7}{|c|}{ MASCULINO } & \multicolumn{6}{|c|}{ FEMENINO } \\
\hline $\mathrm{AÑO}$ & $<14$ & 15-24 & $25-34$ & $35-44$ & 345 & TOTAL & $<14$ & 15-24 & 25-34 & $35-44$ & 345 & Total \\
\hline 1998 & 3 & 24 & 8 & 5 & 3 & 43 & 3 & 35 & 11 & 7 & 2 & 58 \\
\hline 1999 & 2 & 16 & 8 & 7 & 6 & 39 & 2 & 20 & 5 & 10 & 4 & 41 \\
\hline 2000 & 1 & 24 & 15 & 8 & 7 & 55 & 3 & 35 & 9 & 8 & 3 & 58 \\
\hline 2001 & 3 & 25 & 14 & 15 & 2 & 59 & 4 & 31 & 11 & 5 & 3 & 54 \\
\hline 2002 & 3 & 30 & 15 & 11 & 10 & 69 & 5 & 33 & 24 & 7 & 2 & 71 \\
\hline $2003 *$ & 4 & 35 & 11 & 11 & 10 & 71 & 5 & 34 & 26 & 13 & 3 & 81 \\
\hline \multirow[t]{2}{*}{ TOTAL } & 16 & 154 & 71 & 57 & 38 & 336 & 22 & 188 & 86 & 50 & 17 & 363 \\
\hline & \multicolumn{6}{|c|}{$(4.7 \%)(45.8 \%)(21.1 \%)(16.9 \%)(11.3 \%)$} & \multicolumn{6}{|c|}{$(6.1 \%)(51.7 \%)(23.7 \%)(13.7 \%)(4.7 \%)$} \\
\hline
\end{tabular}

* Se ignora la edad de 14 personas de 2003.

Cuadro 4

Distribución del intento de suicidio por mes del año, de 1998 a 2003 en el Hospital General “Agustín O’Horán” de la Secretaria de Salud, en Mérida, Yucatán, México.

\begin{tabular}{|c|c|c|c|c|c|c|c|c|c|c|c|c|c|}
\hline & Ene & Feb & Mar & Abr & May & Jun & Jul & Ago & Sep & Oct & Nov & Dic & Total \\
\hline Año & M F & M F & M F & M F & M F & M F & M F & M F & M F & M F & $\mathrm{M} \mathrm{F}$ & $\mathrm{M} \mathrm{F}$ & M \\
\hline 1998 & 12 & 45 & 65 & 12 & 512 & 34 & 35 & 62 & 25 & 46 & 17 & 73 & 43 \\
\hline 1999 & 37 & 3 & 4 & 2 & 8 & 23 & 3 & 6 & 2 & 4 & 2 & 1 & 39 \\
\hline 2000 & 73 & 5 & 2 & 5 & 63 & 55 & 5 & 6 & 27 & 6 & 6 & 3 & 55 \\
\hline 2001 & 2 & 6 & 4 & 1 & 54 & 85 & 8 & 48 & 144 & 3 & 2 & 5 & 59 \\
\hline 2002 & 7 & 8 & 7 & 5 & 0 & 86 & 47 & 1111 & 63 & 6 & 5 & 2 & 69 \\
\hline 2003 & 97 & 4 & 8 & 8 & 68 & 28 & 103 & 87 & $4 \quad 12$ & 59 & 10 & 5 & 73 \\
\hline Subt & 2931 & 3026 & 2831 & 2227 & 3035 & 2831 & 2331 & 3840 & 3133 & 2835 & $25 \quad 32$ & 2623 & 338375 \\
\hline Total & 60 & 56 & 59 & 49 & 65 & 59 & 54 & 78 & 64 & 63 & 57 & 49 & 713 \\
\hline
\end{tabular}

$\mathrm{M}=$ Masculino $\mathrm{F}=$ Femenino

\section{Cuadro 5}

Mecanismos utilizados para el intento de suicidios registrados de 1998 a 2003 en el Hospital General “Agustín O’Horán” de la Secretaria de Salud, en Mérida, Yucatán, México.

\begin{tabular}{lccccccc}
\hline MECANISMO & $\mathbf{1 9 9 8}$ & $\mathbf{1 9 9 9}$ & $\mathbf{2 0 0 0}$ & $\mathbf{2 0 0 1}$ & $\mathbf{2 0 0 2}$ & $\mathbf{2 0 0 3}$ & TOTAL \\
\hline No reportados & $55(54 \%)$ & $46(57 \%)$ & $50(44.2 \%)$ & $58(51 \%)$ & $51(36 \%)$ & $46(28 \%)$ & $306(42.9 \%)$ \\
Medicamentos & $18(18 \%)$ & $20(25 \%)$ & $27(23.9 \%)$ & $22(19 \%)$ & $43(31 \%)$ & $69(42 \%)$ & $199(27.9 \%)$ \\
Organofosforados & $11(11 \%)$ & $3(4 \%)$ & $9(8 \%)$ & $13(12 \%)$ & $24(17 \%)$ & $24(14 \%)$ & $84(11.8 \%)$ \\
$\begin{array}{l}\text { Ahorcamiento } \\
\text { Arma blanca y }\end{array}$ & $2(2 \%)$ & $1(1 \%)$ & $6(5.3 \%)$ & $4(4 \%)$ & $6(4 \%)$ & $9(5 \%)$ & $28(3.9 \%)$ \\
de fuego & $6(6 \%)$ & $3(4 \%)$ & $6(5.3 \%)$ & $2(2 \%)$ & $1(1 \%)$ & $3(2 \%)$ & $21(2.9 \%)$ \\
Otros & $9(9 \%)$ & $7(9 \%)$ & $15(13.3 \%)$ & $14(12 \%)$ & $15(11 \%)$ & $15(9 \%)$ & $75(10.5 \%)$ \\
Total & $101(100 \%)$ & $80(100 \%)$ & $113(100 \%)$ & $113(100 \%)$ & $140(100 \%)$ & $166(100 \%)$ & $713(100 \%)$ \\
\hline
\end{tabular}

\section{Revista Biomédica}


Prevalencia del intento de suicidio en el Servicio de Urgencias.

Cuadro 6

Fallecimientos registrados a consecuencia del intento de suicidio de 1998 a 2003 en el Hospital General “Agustín O’Horán” de la Secretaria de Salud, en Mérida, Yucatán, México.

\begin{tabular}{lcccc}
\hline A ÑO & Masculino & Femenino & Total de Muertes & Total de Intentos \\
\hline 1998 & 1 & 0 & 1 & 101 \\
1999 & 2 & 0 & 2 & 80 \\
2000 & 3 & 1 & $4(4 \%)$ & 113 \\
2001 & 3 & 0 & $3(3 \%)$ & 113 \\
2002 & 7 & 1 & $8(5.7 \%)$ & 140 \\
2003 & 6 & 1 & $7(4 \%)$ & 166 \\
TOTAL & $22 / 338(6.5 \%)$ & $3 / 375(0.8 \%)$ & $25(3.4 \%)$ & 713 \\
\hline
\end{tabular}

(19.6\%) en 2002 y $166(23.3 \%)$ en 2003, como puede ser observado en la figura 1.

Se obtuvo el total de pacientes atendidos en el área de urgencias de 1999, 2001, 2002 y 2003 para obtener el porcentaje de pacientes por intento de suicidio y se presenta en el cuadro 1 . En cuanto a la distribución del intento de suicidio por género, el $47.4 \%(\mathrm{n}=338)$ fueron hombres y el 52,6\% $(\mathrm{n}=$ 375 ) mujeres, en una proporción de $1: 1$, como puede ser observado en el cuadro 2, (excepto en 1998 y en 2003) y en la figura 2.

Se registraron las edades de las personas que intentaron suicidarse y se agruparon por grupos de 10 en 10 años, encontrando que el rango de edad más afectado es de 15 a 24 años (154 para el sexo masculino y 188 para el sexo femenino), seguido del grupo de 25 a 34 años (71 hombres y 86 mujeres), como puede ser observado en el cuadro 3.

En cuanto a la época o mes del año en la que intentaron suicidarse (cuadro 4), no hubo asociación específica. La mayor frecuencia en relación con el intento suicida fue en el mes de agosto, aunque ésta no tiene significancia estadística.

A pesar de que en la mayoría de los casos no se reportó el mecanismo utilizado para el intento de suicidio, el uso de medicamentos fue el más frecuente (cuadro 5).

En cuanto a las personas que fallecieron a consecuencia del intento de suicidio se registraron sexo, edad y sus mecanismos, en los cuadros 6,7 y 8 .

Se observa en el cuadro 6, que el 3.4\% $(n=25)$ de los que intentaron suicidarse y llegaron al hospital
Cuadro 7

Rango de edades de los fallecidos a consecuencia del intento de suicidio.

\begin{tabular}{cccccc}
\hline AÑO & $15-24$ & $25-34$ & $35-45$ & 345 & TOTAL \\
\hline $\mathbf{1 9 9 8}$ & 1 & 0 & 0 & 0 & 1 \\
$\mathbf{1 9 9 9}$ & 1 & 1 & 0 & 0 & 2 \\
$\mathbf{2 0 0 0}$ & 1 & 2 & 1 & 0 & 4 \\
$\mathbf{2 0 0 1}$ & 1 & 1 & 1 & 0 & 3 \\
$\mathbf{2 0 0 2}$ & 3 & 1 & 2 & 2 & 8 \\
$\mathbf{2 0 0 3}$ & 5 & 0 & 1 & 1 & 7 \\
Total & $12 / 342$ & $5 / 157$ & $5 / 107$ & $3 / 55$ & $25 / 713$ \\
& $(3.5 \%)$ & $(3.2 \%)$ & $(4.6 \%)$ & $(5.4 \%)$ & $(3.5 \%)$ \\
\hline
\end{tabular}

\section{Cuadro 8}

Causa de los fallecimientos a consecuencia del intento de suicidio.

\begin{tabular}{cccccc}
\hline Año & $\begin{array}{c}\text { Organo- } \\
\text { fosforados }\end{array}$ & $\begin{array}{l}\text { Ahorca- } \\
\text { miento }\end{array}$ & $\begin{array}{l}\text { Medica } \\
\text { mentos }\end{array}$ & Otros Total \\
\hline 1998 & 1 & 0 & 0 & 0 & 1 \\
1999 & 0 & 0 & 0 & 2 & 2 \\
2000 & 1 & 2 & 0 & 1 & 4 \\
2001 & 1 & 2 & 0 & 0 & 3 \\
2002 & 2 & 0 & 1 & 5 & 8 \\
2003 & 3 & 1 & 1 & 2 & 7 \\
Total* & $8 / 84$ & $5 / 28$ & $2 / 199$ & 10 & 25 \\
& $(9.5 \%)$ & $(17.8 \%)$ & $(1 \%)$ & & \\
\hline
\end{tabular}

* Número total de defunciones/total de intentos 
S Coronado-Santos, I Diaz-Cervera, L Vargas-Ancona, A Rosado-Franco, A Zapata-Peraza y col.

lo lograron. De los que lo intentaron y lo lograron, se observa que los hombres consiguieron en mayor número consumar el suicidio: $22 / 336$ (6.5\%), que las mujeres: $3 / 363(0.8 \%)(\mathrm{p}<0.05)$, en una proporción 8:1.

De los mecanismos con los que se logró consumar el suicidio, la utilización del ahorcamiento resultó ser el más eficaz, $(5 / 28,17.8 \%)$, seguido de la ingestión de algún compuesto organofosforado (8/ $84,9.5 \%)$.

\section{DISCUSIÓN.}

En nuestro país el suicidio y sus variantes se han convertido en los últimos años en un verdadero problema de salud pública, debido a que la tasa de suicidio ha ido en aumento año con año para uno y otro sexo. De 1970 a 1994 la tasa pasó de 1.13 a 2.89 por 100,000 habitantes; durante el año 2000 ocurrieron en México 429 intentos de suicidio, de los cuales el $43.8 \%$ fue realizado por hombres $(n=188)$, y el restante $56.2 \%$ por mujeres $(n=241)$, lo cual es estadísticamente significativo a favor del sexo femenino $(p=0.0108)$. Cabe señalar que mientras más mujeres intentan suicidarse, son más los hombres que realmente lo hacen $(5,6)$.

De acuerdo a los resultados obtenidos podemos concluir que el intento de suicidio es un motivo frecuente de consulta en el servicio de urgencias del Hospital General "Agustín O’Horán" de la ciudad de Mérida, Yucatán, México. Asimismo, se observó que el número de intentos de suicidio fue aumentando año con año, tanto en el número de casos como en el porcentaje que representan para el total de la consulta de urgencias: ( $0.93 \%$ de casos registrados, respecto al del total de consultas en 1999 y 2.4\% en el 2003). Otro dato encontrado, es que a diferencia de lo reportado en la literatura, la relación hombre-mujer, en cuanto al intento de suicidio, es de 1:1. (47.4\% de hombres vs 52,6\% mujeres. Las edades en las que se presentó con más frecuencia el intento de suicidio de este estudio, son entre los 15 y 24 años, tanto para el sexo femenino como para el masculino, siguiéndole en frecuencia el rango de edad de 25 a 34 años. Estos datos concuerdan con la literatura mundial
(5).

Se observó que no existe una época o mes del año en el que aumente la frecuencia del número de intentos de suicidio, aunque en el mes de agosto se observó un ligero aumento para uno y otro sexo, pero no resultó significativo.

La proporción de fallecimientos a consecuencia del intento de suicidio coincide con la que se reporta en la literatura: fue mayor el número de hombres que lograron su objetivo, en relación a las mujeres, en proporción 8:1. También pudo observarse que el número de fallecimientos por intento de suicidio que llegaron al hospital fue en aumento, ya que en 1998 se registró un fallecimiento y en 2003 se registraron 7.

Puede onservarse que aunque la mayoría de estos pacientes tenían estre 15 y 24 años hubo mayor número de defunciones en los grupos de 35 a 45 y mayor de 45 años.

Llama la atención que de los mecanismos más utilizados para lograr consumar el suicidio, la intoxicación por organofosforados fue el principal, seguido del ahorcamiento. Esto al parecer es diferente a otros estudios de la República Mexicana, en donde el segundo predomina (6). Los resultados muestran que el ahorcamiento fue el más efectivo método de suicidio.

Hacemos notar que tanto los intentos de suicidio como los suicidios son competencia del ministerio público, y por ello no obtuvimos la información de manera precisa. Además, en muchos casos de intento de suicidio, no se integró expediente porque no ingresaron los pacientes $\mathrm{y}$, por lo tanto, no se registraron, lo que ocasiona un subregistro.

Es importante comparar el número de casos de intento de suicidio del Hospital O'Horán con las cifras reportadas por el Instituto Nacional de Estadística, Geográfica e Informática (INEGI): en 1998 el O'Horán reportó 101 intentos de suicidio, 80 en 1999; 113 en el 2000; 113 en el 2001; el INEGI en estos mismos años reportó 124 en 1998; 110 en 1999; 109 en el 2000 y 115 en el 2001. Esto confirma que hay un subregistro ya que el INEGI reporta el total de intentos de suicidio del estado de Yucatán, y hay que tomar en cuenta la existencia de tres hospitales

\section{Revista Biomédica}


generales y uno de psiquiatría, más el hospital O’Horán que por sí solo genera el dato total reportado por el INEGI.

\section{REFERENCIAS.}

1.- Baquedano G. Programa universitario para el estudio del suicidio. Facultad de Ciencias Antropológicas, Universidad Autónoma de Yucatán. 2002. Disponible en: http:// members.tripod.com/-baquedano/index-9.html.

2.- Alan A. Correlation of suicidal and violent behavior in different diagnostic categories in hospitalized adolescent patients. Am J Acad Child Adol Psychiat 1995; 34: 7-15.

3.- Bonger B. Suicide guidelines for assessment, management and treatment. Oxford: Blackwell Scientific Publications; 1992.

4.- Beck AT, Brown G, Berchick RJ. Hopelessness and eventual suicide; a year prospective study of patients hospitalized with suicidal ideation: Am J Psychiat 1985; 5:1427.

5.- Rich CL, Young D, Fowler RC. San Diego suicide study I. Young vs old subjects. Arch Gen Psychiat 1996; 43: 577-82.

6.- Borges G, Rosovsky H, Gómez C, Gutiérrez R. Epidemiología del suicidio en México de 1970 a 1994; Salud Pública Mex 1996; 38:197-206.

7.- Shaffer D, Piacentini J. Suicide and attempted suicide in: Rutter M, Taylor E, Hersov L, Eds. Child and Adolescent Psychiatry. Oxford: Blackwell Scientific Publications 1994; p. 407-24.

8.- Ulloa-Fresia Ch. Tentativas y consumación de suicido en niños y adolescentes. Rev Chil Pediatr 1993; 64: 272-6. 